\section{A New Look at Three Potential Mechanisms Proposed for the Carcinogenesis of $5 \mathrm{G}$ Radiation}

\author{
Bevelacqua J. J. ${ }^{1 \oplus}$, Mehdizadeh A. R. ${ }^{2}$, Mortazavi S. \\ M. J. ${ }^{2,3 * \odot}$
}

I $\mathrm{n}$ telecommunications, the fifth generation $(5 \mathrm{G})$ is the new technology standard for cellular networks. However, the potential hazards of this telecommunication technology for human health and the environment have not yet been fully investigated by scientists independent from industry. It is believed that the widespread usage of $5 \mathrm{G}$ technology, can lead to significant increases in human exposure to radiofrequency electromagnetic fields (RF-EMF). Given this consideration, in an appeal to the European Union (EU), more than 180 scientists and physicians from 36 countries have warned about the potential dangers of $5 \mathrm{G}$ technology [1]. Dr. Lennart Hardell, Professor of Oncology at Örebro University in Sweden and one of the initiators, states: "The telecom industry is trying to roll out technology that may have very real, unintended harmful consequences..... We are very concerned that the increase in radiation exposure by $5 G$ leads to damage that cannot be reversed" [1]. While such a large number of experts from different countries have signed this appeal, it is very interesting that an author that is not independent from the telecommunication industry, claims that only a few people believe $5 \mathrm{G}$ has adverse health effects "Beyond this consideration and responding to some unfounded concerns, the paper reaffirms that $5 G$ will not have the negative effect on people's health about which a few individuals have speculated" [2]. Hardell and Carlberg in their recent publication have addressed their concerns over studies with ties to industry "Conflicts of interest and ties to the industry seem to have contributed to the biased reports" [3]. Hardell and Carlberg have also criticized the EU for not acknowledging an appeal to the EU that is currently endorsed by more than 390 scientists and medical doctors requesting a moratorium on $5 \mathrm{G}$ deployment until proper scientific evaluation of potential adverse health effects has been conducted [3]. Exposure to high levels of radiofrequency electromagnetic fields has been proven to be detrimental for humans and the environment. Despite a large body of evidence, there are still scientists [4] who claim that there is no scientific evidence supporting a potential link between $5 \mathrm{G}$ and the risk of malignancies such as skin cancer "Concerns have been raised on online fora and in scientific literature regarding a link between $5 G$
${ }^{1} \mathrm{PhD}$, Bevelacqua Resources, Richland, WA 99352, USA

${ }^{2} \mathrm{PhD}$, Department of

Medical Physics and

Engineering, School of

Medicine, Shiraz Univer-

sity of Medical Sciences,

Shiraz, Iran

${ }^{3} \mathrm{PhD}$, lonizing and

Non-ionizing Radiation

Protection Research

Center (INIRPRC), Shiraz

University of Medical Sci

ences, Shiraz, Iran

*Corresponding author: S. M. J. Mortazavi Department of Medical Physics, School of Medicine, Shiraz University of Medical Sciences,

Shiraz, Iran

E-mail: mortazavismj@ gmail.com

Received: 13 August 2020 Accepted: 20 August 2020 
Bevelacqua J. J. Mehdizadeh A. R., Mortazavi S. M. J.

and skin cancer [5], despite an absence of scientific evidence" [6]. It is indeed surprising that Rafferty et al., have cited a report published in the New York Times (NYT) to support their claim about a paper by Mehdizadeh and Mortazavi that addresses the theoretical basis of potential association of $5 \mathrm{G}$ and skin cancer. This NYT report has been seriously criticized by Mehdizadeh and Mortazavi, as follows: "Unfortunately, the approach of New York Times in this report is not scientific. William J. Broad in his report published July 16, 2019 criticized Dr. Bill P Curry for not considering the so-called "protective effect of human skin". Some recent publications have tried to convince the readers that current concerns about 5G high frequencies are not real. In some cases, such as the paper by Rafferty et al., numerous major shortcomings and the lack of expertise of the authors in physics and biology of RF-EMFs, suggests that these papers, deserve retraction.

Kostoff et al., [7] in their paper published recently, state: "The common 'wisdom' presented in the literature and media is that, if there are adverse impacts resulting from high-band $5 G$, the main impacts will be focused on near-surface phenomena, such as skin cancer, cataracts, and other skin conditions. However, there is evidence that biological responses to millimeter-wave irradiation can be initiated within the skin, and the subsequent systemic signaling in the skin can result in physiological effects on the nervous system, heart, and immune system" [8].

\section{Current Theories about the Carcinogenesis of $5 \mathrm{G}$}

The first model of carcinogenesis of 5G, was developed by Mehdizadeh and Mortazavi and as shown in Figure 1, high-frequency $5 \mathrm{G}$ radiation penetrates living skin cells and can damage them severely due to its low penetration and very high energy deposition per unit distance below the skin surface [5]. Given this consideration, absorption of $5 \mathrm{G}$ radiation in skin can lead to the generation of high levels of free radicals, which in turn increases the risk of skin cancer. Yakymenko et al., have reported that among 100 peer-reviewed publications on oxidative effects of low-intensity radiofrequency radiation included in their review, 93 studies showed that radiofrequency radiation induced oxidative effects in biological systems [9].

Oxidative stress that is caused by the increase in free radicals including reactive oxygen species (ROS) can play a basic role in pathological conditions of diseases such as cancer [10-12]. As reported by Singh et al., free radicals are involved in the pathogenesis of a multistage process of car-

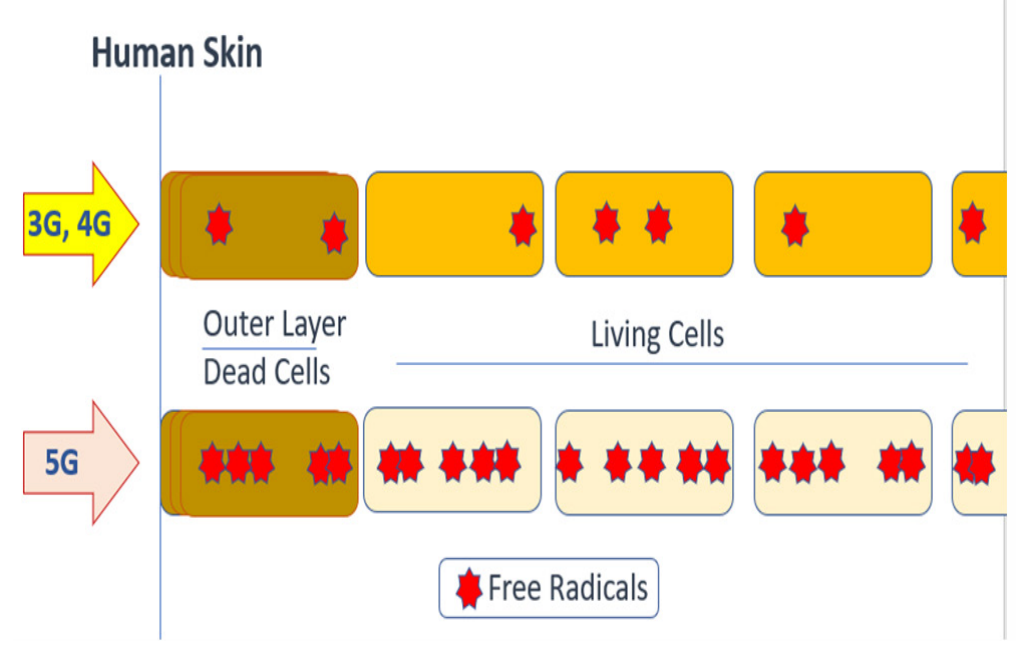

Figure 1: Due to its low penetration and very high energy deposition per unit distance below the skin surface, high-frequency $5 \mathrm{G}$ radiation penetrates and severely damages living skin cells. 
cinogenesis [13]. Free radicals are believed to cause DNA base damages, strand breaks, damage to the tumor suppressor genes, and an increased expression of the protooncogenes. Moreover, DNA damages induced by reactive oxygen species (ROS) may contribute to higher rates of mutation, genome instability, apoptosis, associated tissue regeneration, and cell proliferation [13]. Lobo et al., introduce cancer as a "free radical" disease "Cancer and atherosclerosis, two major causes of death, are salient "free radical" diseases" [14]. Although oxidative stress and potential DNA damages do not necessarily increase the chance of cancer, as addressed by Kostoff et al., a rise in near-surface phenomena, such as skin cancer and cataracts, can be expected [7].

Furthermore, Betzalel et al., have previously developed a simulation model of human skin. It focuses on the multi-layer structure of skin, and especially on the helical segment of the sweat duct that serves as an antenna leading to high specific absorption rate (SAR) of the skin at extremely high frequencies, where resonances of the sweat duct-short wavelength radiation occur. Based on their modeling results, they state: "There is enough evidence to suggest that the combination of the helical sweat duct and wavelengths approaching the dimensions of skin layers could lead to non-thermal biological effects. Such fears should be investigated and these concerns should also [a]ffect the definition of standards for the application of $5 G$ communications" [15]. A paper by Tripathi et al., [16] examined in detail the morphology of human sweat ducts observed by optical coherence tomography. Ref. 16 noted that their frequency of resonance lies in the terahertz region. Given the range of duct sizes and wavelength of the $5 \mathrm{G}$ radiation, the importance of the shorter $5 \mathrm{G}$ wavelengths becomes apparent. This is supported by Tripathi et al., suggesting that resonances occur in the sweat ducts at $\mathrm{THz}$ frequencies. As the wavelength of the nonionizing radiation decreases, the resonance coupling and energy deposition will increase. This phenomenon is illustrated in Figure 1.

The importance of the coupling and $5 \mathrm{G}$ energy deposition is a complex process. It depends on the incident power density, particular 5G frequency, and the absorption coefficient for the biological medium. Determination of the absorption coefficient is also complex and depends on a number of factors including the angular frequency of the $5 \mathrm{G}$ radiation, the conductivity of the tissue of interest, permittivity of the medium, relative dielectric constant, and permeability of the medium [17].

As noted in our discussion the detriment caused by $5 \mathrm{G}$ radiation cannot be dismissed without a thorough evaluation of the tissue at risk as well as the energy absorption. This uncertainty suggests that further study is warranted and should consider the mechanisms proposed in this paper.

\section{Conflict of Interest}

None

\section{References}

1. EU 5G Appeal - Scientists warn of potential serious health effects of 5G. [cited 2020 July 19] Available from: https://www.jrseco.com/european-union-5g-appeal-scientists-warn-of-potential-serious-health-effects-of- $5 \mathrm{~g} /$.

2. Soldani D. Fighting Pandemics By Exploiting 5G, Al and Bigdata Enabled Technologies: How $5 \mathrm{G}$ could help us to stem Covid-19 outbreaks. Journal of Telecommunications and the Digital Economy. 2020;8(2):146-58. doi: 10.18080/jtde.v8n2.257.

3. Hardell L, Carlberg M. [Comment] Health risks from radiofrequency radiation, including $5 \mathrm{G}$, should be assessed by experts with no conflicts of interest. Oncology Letters. 2020;20(4):1. doi: 10.3892/0l.2020.11876.

4. Rafferty S, O'Connor C, Murphy M. "Fake News'-5G mobile phones and skin cancer: A global analysis of concerns on social media. Skin Research and Technology. 2020. doi: 10.1111/srt.12912. PubMed PMID: 32674224.

5. Mehdizadeh AR, Mortazavi SMJ. 5G Technology: Why Should We Expect a shift from RF-Induced Brain Cancers to Skin Cancers?. J Biomed Phys Eng. 2019;9(5): 505-6. doi: 10.31661/jbpe.v0i0.1225. PubMed PMID: 31750263. 
Bevelacqua J. J. Mehdizadeh A. R., Mortazavi S. M. J.

PubMed PMCID: PMC6820018.

6. Broad WJ. The 5G health hazard that Isn't. The New York Times; 2019. Available from: https://www. nytimes. com/2019/07/16/science/5g-cellphones-wireless-cancer. html.

7. Kostoff RN, Heroux P, Aschner M, Tsatsakis A. Adverse health effects of $5 G$ mobile networking technology under real-life conditions. Toxicology Letters. 2020;323:35-40. doi: 10.1016/j.toxlet.2020.01.020. PubMed PMID: 31991167.

8. Russell CL. 5 G wireless telecommunications expansion: Public health and environmental implications. EnvironMental Research. 2018;165:484-95. doi: 10.1016/j.envres.2018.01.016. PMID: 29655646.

9. Yakymenko I, Tsybulin 0, Sidorik E, Henshel D, Kyrylenko 0, Kyrylenko S. Oxidative mechanisms of biological activity of low-intensity radiofrequency radiation. Electromagnetic Biology and Medicine. 2016;35(2):186-202. doi: 10.3109/15368378.2015.1043557. PubMed PMID: 26151230.

10. Klaunig JE, Kamendulis LM. The role of oxidative stress in carcinogenesis. Annu. Rev. Pharmacol. Toxicol. 2004;44:239-67. doi: 10.1146/annurev.pharmtox.44.101802.121851. PubMed PMID: 14744246.

11. Nathan C, Ding A. SnapShot: reactive oxygen intermediates (ROI). Cell. 2010;140(6):951. doi: 10.1016/j. cell.2010.03.008. PubMed PMID: 20303882.

12. Pham-Huy LA, He H, Pham-Huy C. Free radicals, antioxidants in disease and health. International Journal of BioMedical Science: IJBS. 2008;4(2):89-96. PubMed PMID: 23675073. PubMed PMCID: PMC3614697.

13. Singh AK, Pandey P, Tewari M, Pandey HP, Gambhir IS, Shukla HS. Free radicals hasten head and neck cancer risk: A study of total oxidant, total antioxidant, DNA damage, and histological grade. Journal of Postgraduate Medicine. 2016;62(2):96-101. doi: 10.4103/0022-3859.180555. PubMed PMID: 27089108. PubMed PMCID: PMC4944358.

14. Lobo V, Patil A, Phatak A, Chandra N. Free radicals, antioxidants and functional foods: Impact on human health. Pharmacognosy Reviews. 2010;4(8):118-26. doi: 10.4103/0973-7847.70902. PubMed PMID: 22228951. PubMed PMCID: PMC3249911.

15. Betzalel N, Ishai PB, Feldman Y. The human skin as a sub-THz receiver-Does $5 \mathrm{G}$ pose a danger to it or not? Environmental Research. 2018;163:208-16. doi: 10.1016/j.envres.2018.01.032. PubMed PMID: 29459303.

16. Tripathi SR, Miyata E, Ishai PB, Kawase K. Morphology of human sweat ducts observed by optical coherence tomography and their frequency of resonance in the terahertz frequency region. Scientific Reports. 2015;5:9071. doi: 10.1038/srep09071.

17. Bevelacqua JJ. Contemporary health physics: problems and solutions. John Wiley \& Sons; 2009. 